

\title{
Commentary
}

\section{What's in a name? The confusion in nomenclature of low-acuity specialist roles in paramedicine}

David N Long, PhD candidate'; Michele Clark BOccThy[Hons], BA, PhD, Professor and Post-graduate Coordinator ${ }^{1}$; David Lim, DrPH, Lecturer ${ }^{1}$; Scott Devenish, PhD, Director of International Engagement and Recruitment ${ }^{1}$

\begin{abstract}
Affiliation:
${ }^{1}$ School of Clinical Sciences, Queensland University of Technology, Brisbane

Corresponding Author: David Nicholas Long, d5.long@hdr.qut.edu.au

Paramedics are increasingly seen as an integral component of the healthcare continuum (1). Whilst the core functions of paramedic services to treat and transport the sick and injured to hospital have remained largely unchanged, a significant paradigm shift in the care of non-urgent, low-acuity patients has been gathering momentum over the last two decades (2). Paramedic services across North America, the United Kingdom and Australasia have progressively embraced new paradigms of care in the out-of-hospital setting to provide alternate pathways for low-acuity patients other than to the emergency department. However, the current nomenclature to define paramedics who specialise in the low-acuity setting is confusing, inconsistent and arguably misrepresentative. Most notable is the popularity in the literature of the umbrella term 'community paramedicine' for all 'non-traditional' paramedics engaged in the low-acuity setting.
\end{abstract}

The definition of community paramedicine proposed by the International Roundtable on Community Paramedicine (IRCP) appears to be gaining traction around the world $(3,4)$ :

'Community paramedicine is a model of care whereby paramedics apply their training and skills in 'non-traditional' community-based environments, often outside the usual emergency response and transportation model. The community paramedic practices within an 'expanded scope', which includes the application of specialised skills and protocols beyond the base paramedic training. The community paramedic engages in an 'expanded role' working in non-traditional roles using existing skills (5 p2).'

The above definition however does not articulate the important philosophical differences in distinguishing other models of care that lie along the spectra of low-acuity service delivery in paramedicine. Indeed, only recently has a theoretical framework been applied to describe the conceptual basis of a community paramedic model of care (6). Despite being an early contribution to the field, the model may not be transferable to other low-acuity models due to variances in such areas as education, scope of practice and perhaps most significantly, local community needs.

We contend a more fundamental and simple classification of low-acuity service delivery models into two discernible paradigms is required to limit the rapidly expanding nomenclature in community paramedicine. One paradigm is aligned with a 'preventative/follow-up' model of care to reduce the incidence of unscheduled/emergent care by working collaboratively with physicians and other primary care services in areas such as chronic disease management, health promotion/education and early intervention (1). The common descriptor for a paramedic employed in this model of care is 'Community Paramedic' - and more recently updated to 'Community Paramedicine Provider' for those employed in the Mobile Integrated Health Care model in the United States (7). 
The other paradigm is a 'reactive' model in which paramedics respond to calls for unscheduled care and are usually dispatched by ambulance control centres through traditional notification systems such as calls to '000' in Australia or ' 999 ' in the United Kingdom. The patient is treated in their own residence for most often, a low-acuity presentation and if necessary, referred for follow-up care which is likely to be with the patient's general practitioner. Common descriptors include 'Extended Care Paramedic', 'Paramedic Practitioner' and 'Emergency Care Paramedic' and are most commonly employed in Australasia and the United Kingdom.

Although the delineation between paradigms is ambiguous at times, the commonality between both models of care has been in the specialisation of paramedics to service local health care needs and to better integrate paramedic services into the healthcare continuum (3). Nevertheless, given the two distinct models of care, ie. preventative/follow-up and reactive, the current umbrella term of 'community paramedicine' - and by extension the role descriptor 'Community Paramedic' - does not accurately reflect the philosophical differences between low-acuity services provided by paramedics. Thus an Extended Care Paramedic in an Australian jurisdiction is not a Community Paramedic in the sense of their North American counterparts.

Representative bodies for paramedics in the United Kingdom (8) and Australia (9), in which 'reactive' models of low-acuity service delivery are most commonly employed, have also made forays into nomenclature favouring the term 'specialist' in either job title or role description. In keeping with local naming conventions, this commentary proposes the introduction of the single term 'Specialist Paramedic - Community' to differentiate paramedics employed in a 'reactive' model of low-acuity service delivery from 'Community Paramedics' employed in a 'preventative/follow-up' model of service delivery. In all likelihood, the term 'community paramedicine' will be popularly accepted as the umbrella term for both models of low-acuity service delivery, hence the inclusion of the term 'Community' as an adjunct to the primary classification of 'Specialist Paramedic'.

Paramedicine is in a state of rapid transition in order to complement service delivery models that are more innovative, equitable and able to meet the current and future needs of the community. However the nomenclature has not kept pace with the transition evidenced by the current terminology which does not sufficiently capture the roles and responsibilities of paramedics employed in non-traditional positions. The time is ripe for a consensus in nomenclature that transcends international boundaries with consistency in nomenclature, a hallmark of the evolving professionalism in paramedicine. Moreover, any move towards national registration will make clarity around nomenclature more pressing as growing professionalisation will invariably see a growth in specialisation. Thus consistency, appropriateness and clarity of nomenclature will move from academic debate to one of practical and professional necessity.

\section{References}

1. Cooper SJ, Grant J. New and emerging roles in out of hospital emergency care: a review of the international literature. Int Emerg Nurs 2009;17:90-8. DOI:10.1016/j.ienj.2008.11.004

2. Catterall M. The role of paramedics with extended practice: exploring the healthcare context. Journal of Paramedic Practice 2012;4:1.

3. Bigham BL, Kennedy SM, Drennan I, Morrison LJ. Expanding paramedic scope of practice in the community: a systematic review of the literature. Prehosp Emerg Care 2013;17:361-72. DOI:10.3109/10903127.2013.792890

4. O'Meara P. Community paramedics: a scoping review of their emergence and potential impact. International Paramedic Practice 2014;4(1):5-12. DOl:http://dx.doi.org/10.12968/ippr.2014.4.1.5

5. Community Paramedicine Submission to the Standing Committee on Health. Emergency Medical Services Chiefs of Canada; 2012. 13. Available at: http://ircp.info/Portals/11/Downloads/Policy/2012\%20EMSCC\%20CP\%20 Submission $\% 20$ to\%20Canadian\%20Parliament.pdf

6. O'Meara P, Stirling C, Ruest M, Martin A. Community paramedicine model of care: an observational, ethnographic case study. BMC Health Serv Res 2016;16:1-11. DOI: http://dx.doi.org/10.1186/s12913-016-1282-0

7. Choi B, Blumberg C, Williams K. Mobile Integrated Health Care and Community Paramedicine: An Emerging Emergency Medical Services Concept. Ann Emerg Med 2016;67:361-6. DOl:http://dx.doi.org/10.1016/j.annemergmed.2015.06.005

8. Paramedic Career Framework. Third edition. College of Paramedics; 2015. 48. Available at: www.collegeofparamedics. co.uk/downloads/Post-Reg_Career_Framework_3rd_Edition.pdf

9. Paramedicine Role Descriptions. Paramedics Australasia; 2012. 23. Available at: www.paramedics.org/content/2009/07/ PRD_211212_WEBONLY.pdf 